\title{
HALO FORMATION AND CONTROL
}

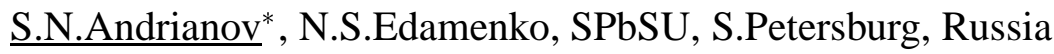

\begin{abstract}
In this report we suggest some approaches to very intricate problem of the halo formation process. It is known that this process leads to the formation of a sufficiently compact core and a spreading cloud - halo, which surrounds the core. Our approach to this problem is based on two main objects: initial distribution functions and matrix formalism for Lie algebraic tools for time evolution of particle beam. Usage of the matrix formalism allows to investigate the influence of different forms of starting model distributions. All calculations are based on symbolic representation of necessary mapping generated by space charge forces and external control fields. This allows us to formulate the basic requirements which are necessary for halo formation, that gives us a possibility to control this process.
\end{abstract}

\section{INTRODUCTION}

It is known that usually the evaluation of space charge effects on the beam dynamics requires intensive numerical calculation. That is why there are innumerable publications, which devoted to modeling of space charge dynamics for concrete machines. The most of papers concentrate upon an numerical analysis of the influence of the beam line characteristics for matched or/and unmatched beams. Nevertheless the problem of the influence of beam distribition characteristics keep through our study. But it is impossible to obtain total presentation of, for example, halo problem without thorough investigation of different kind of conditions which affect halo formation. Most recent publications have dealt with either the KV-distribution or several simple distributions. In the paper [1] a very interesting approach to halo production is presented. The authors suggest new concepts for halo description which allows, in particular, to solve problems of halo control. All approaches to this problem have ultimatelly depended upon the calculation techniques that can be applied. To understand halo formation process, in this work we consider the evalution of the phase-space distribution in terms of matrix representation for Lie transformations [2]. This allows us to use computer algebra methods and codes to reduce the real time needed for the numerical calculations. The external forces are assumed radial and periodical, as in solenoid channels. This force model can be also appleid to quadrupole focusing if the phase advance is not too large. The focusing force as the space-charge forces are considered upto aberrations of the third order. In this paper we use the model of long beams with an elliptical cross-section in the transverse phase-space. Some models of phase-space distri-

\footnotetext{
*Serge.Andrianov@pobox.spbu.ru
}

bution functions are described [3]. The Ferrer's integrals technique is used for calculation of space-charge forces in a symbolic form. Note if we neglect space-charge forces the motion equation for Lie transformation have linear operator form, but if the space-charge forces are included in our investigation the corresponding equation become nonlinear as the generating vector field depends on beam characteristics. Nonlinear nature of these equations leads to a necessity to use the successive approximations method. Obtained convergence conditions and algorithms give opportunity to estimate a current step value in advance and to create necessary software for modeling [4]. The truncated matrix equations (up to third order) are solved with the use of the matrix formalism for Lie algebraic tools with necessary symplification procedure [5]. As a result of this work, there are a number of computer experiments that show us what kind of both beam and beamline characteristics have to be taken in account. The necessary software was created using the dynamic modeling paradigm [6].

\section{A SPACE CHARGE DESCRIPTION}

\subsection{The Initial Space Charge Distributions}

The initial space-charge distribution in the phase-space can be written in the general form $f_{0}(X)=\sum_{k=0}^{\infty} f_{k}^{0} X^{[k]}$ or in the case of elliptical symmetry

$$
f_{0}(X)=\sum_{k=0}^{\infty} a_{k}^{0} \kappa^{2 k}=\sum_{k=0}^{\infty} a_{k}^{0}\left(X^{[k]}\right)^{*} \mathbf{A}_{0}^{\{k\}} X^{[k]},
$$

where $X^{[k]}=\underbrace{X \otimes \cdot \ldots \cdot \otimes X}_{k-\text { times }}$ is the Kronecker power of the phase vector $X=\left(x, p_{x}, y, p_{y}\right)^{*}, \operatorname{dim} X^{[k]}=$ $\left(\begin{array}{c}k+3 \\ k\end{array}\right), \mathbf{A}_{0}^{\{k\}}$ is the symmetrical Kronecker power of the initial form matrix $\mathbf{A}_{0}: \kappa^{2}=X^{*} \mathbf{A}_{0} X$ :

$$
\begin{aligned}
& \left(\mathbf{A}^{\{k\}}\right)_{i l}=b_{i}(k)\left(\mathbf{A}_{0}^{[k]}\right)_{i l}, \\
& i, l=\overline{1,\left(\begin{array}{c}
n+k-1 \\
k
\end{array}\right)}, \quad b_{i}(k)=k ! / k_{1} ! \ldots k_{n} ! .
\end{aligned}
$$

\subsection{The Self-Field of the Space Charge}

Using the Ferrers's integrals technique we calculate the desired space-charge forces in symbolic forms for some modelfunction of space-charge distributions. In particular, we obtain the components of the vector of self-electrical field in the form

$$
E_{\xi, \eta}=E_{\xi, \eta}^{0}+\triangle E_{\xi, \eta}, \quad E_{\xi}^{0}=\frac{4 \pi \rho_{0}}{\epsilon_{0}} \frac{a b}{a(a+b)} \xi,
$$




$$
E_{\eta}^{0}=\frac{4 \pi \rho_{0}}{\epsilon_{0}} \frac{a b}{b(a+b)} \eta .
$$

where $\xi, \eta$ are local coordinates in which the transverse cross-section has the form of a canonical ellipse. The values of $\Delta E_{\xi}, \triangle E_{\eta}$ are calculated with the help of $M A P L E$ codes. Note that for the KV distribution we have $\triangle E_{\xi}=$ $\triangle E_{\eta}=0$. Besides, if the arbitrary distribution $\rho(x, y)=$ $\rho_{0} \Phi\left(\kappa_{1}^{2}\right)$ is a polynomial of $n$-th order with respect to the variables $\kappa_{1}^{2}$ the functions $\triangle E_{\xi}$ and $\triangle E_{\eta}$ are polynomials of $(2 n+1)$-th order with respect to the variables $\xi$ and $\eta$. Then we can return to the coordinates $x, y$ referenced to a beamline system.

\section{THE MOTION EQUATION}

\subsection{The Motion Equation for a Particle}

For a nonbunched beam (the longitudinal self-electric field is missing) the motion equation for single particle can be written in the following matrix form

$$
\frac{d X}{d s}=\sum_{k=1}^{\infty}\left\{\mathbf{P}_{e x t}^{1 k}(s)+\mathbf{P}_{s e l f}^{1 k}(s)\right\} X^{[k]} .
$$

The matrices $\mathbf{P}_{e x t}^{1 k}$ and $\mathbf{P}_{\text {self }}^{1 k}$ describe the external and space-charge fields correspondingly [4].

\subsection{Transfer Map in the Presence of the Space- Charge}

It is known that the Lie algebraic tools is very valuable tools to studying beam dynamics without space-charge. The Lie map satisfies to the following linear operator equation

$$
\frac{d \mathcal{M}\left(s \mid s_{0}\right)}{d s}=\mathcal{L} \circ \mathcal{M}\left(s \mid s_{0}\right),
$$

where $\mathcal{M}\left(s \mid s_{0}\right)$ is a time-displacement operator (Lie map) between moments $s_{0}$ and $s$ :

$$
\mathcal{M}: X_{0} \rightarrow X=\mathcal{M}\left(s \mid s_{0}\right) \cdot X_{0} .
$$

and $\mathcal{L}$ is a Lie operator associated with a generating vector field. If the beam is an ensemble of noninteracting particles then the operator $\mathcal{L}$ depend only on beamline parameters. If we have to take into account the space-charge forces the Lie operator will depend on beam characteristics and as result it will depend on the Lie map. In this case we use nonlinear motion equation for Lie map already:

$$
\frac{d \mathcal{M}\left(s \mid s_{0}\right)}{d s}=\mathcal{L}(\mathcal{M}) \circ \mathcal{M}\left(s \mid s_{0}\right) .
$$

In the frame of the matrix formalism [2] we can represent this map in the form $\mathcal{M}: X_{0} \rightarrow X=\mathcal{M}\left(s \mid s_{0}\right) \cdot X_{0}$,

\subsection{The Matrix Formalism for Lie Map}

According to the matrix formalism [2] we can write

$$
X=\mathcal{M}\left(s \mid s_{0}\right) \cdot X_{0}=\sum_{k=1}^{\infty} \mathbf{M}^{1 k}\left(s \mid s_{0}\right) X_{0}^{[k]},
$$

where $\mathbf{M}^{1 k}$ are matrices which can be calculated with the help of the matrix formalism tools.

According to our suggestions the beam particles occupy some elliptical cross-section $\mathfrak{M}_{0}$ in an initial state:

$$
\mathfrak{M}_{0}=\left\{X_{0}: X_{0}^{*} \mathbf{A}_{0}^{11} X_{0} \leq 1\right\}
$$

As we mention above in the presence of the space-charge the motion equation for the map $\mathcal{M}$ assume a nonlinear form. For the solution of this equation in this paper we propose the method of step-by-step approximations. The basic idea of this method in our case is to calculate the distribution function according to the algorithm described in [4]).

From the known properties of Lie maps we can write for an arbitrary function of an initial distribution $f_{0}(X)=$ $f\left(X, s_{0}\right): f(X, s)=f_{0}\left(\mathcal{M}^{-1}\left(s \mid s_{0}\right) \cdot X\right)$. In our case we have $\mathcal{M}^{-1}\left(s \mid s_{0}\right) \cdot X=\sum_{k=0}^{\infty} \mathbf{T}^{1 k}\left(s \mid s_{0}\right) X^{[k]}$, where $\mathbf{T}^{10}=-\mathbf{M}^{10}, \mathbf{T}^{11}=\left(\mathbf{M}^{11}\right)^{-1}$ and other matrices $\mathbf{T}^{1 k}$ for $k>1$ can be calculated with the help of the recurrent generalized Gauss's algorithm using the matrices $\mathbf{M}^{1 k}$. It is worthy to note that according to this algorithm one should inverse only the matrix $\mathbf{M}^{11}$ and then use only matrix operations for calculation the necessary matrices $\mathbf{T}^{1 k}$ up to the desired order. So after some calculations we can obtain the following equation

$$
\begin{gathered}
f(X, s)=f_{0}\left(\mathcal{M}^{-1} \cdot X\right)= \\
a_{0}^{0} \sum_{k=1}^{\infty} a_{k}^{0}\left(\mathcal{M}^{-1} \cdot X^{[k]}\right)^{*} \mathbf{A}_{0}^{\{k\}}\left(\mathcal{M}^{-1} \cdot X^{[k]}\right)= \\
a_{0}^{0}+\sum_{k=1}^{\infty} a_{k}^{0} \sum_{l=k}^{\infty} \sum_{j=k}^{\infty}\left(X^{[l]}\right)^{*} \mathbf{B}_{k}^{l j} X^{[j]} \\
\mathbf{B}_{k}^{l j}=\left(\mathbf{T}^{k l}\right)^{*} \mathbf{A}_{0}^{\{k\}} \mathbf{T}^{k j} .
\end{gathered}
$$

For the test of the convergence of our approximations methods the following condition can be used

$$
\begin{aligned}
& \left\|f\left(\mathcal{M}_{k+1}^{-1} \circ X\right)-f\left(\mathcal{M}_{k}^{-1} \circ X\right)\right\| \leq \\
& \leq \beta\left\|f\left(\mathcal{M}_{k}^{-1} \circ X\right)-f\left(\mathcal{M}_{k-1}^{-1} \circ X\right)\right\|
\end{aligned}
$$

for $\beta<1, k$-is an iterative loop number. The constant of this method $\beta$ can be calculated as a function of the initial beam characteristics and the beamlines parameters. The condition $\beta<1$ allows the limitations on the step values $\left|s_{k}-s_{k-1}\right|=\triangle s_{k}$ to be calculated which guarantee the fulfilment of the inequality $\beta<1$.

\section{COMPUTER EXPERIMENTS}

The approach discussed above was used for some practical problems: the halo formation problems. The corresponding computer experiments was developed both in symbolic (with the help of MAPLE codes) and in numerical modes. 
The symbolic investigation was carried out with main purpose to understand what parameters have the sufficient influence on halo production. For this task we studied the images of an initial beam state (in the distribution function terms) and used the concepts of virtual scrapers for investigation of what parts of the initial beam give the basic contribution to halo formation. According to this approach we transform the aperture boundaries of virtual scrapers with the help of inverse maps from some current sections to the initial point. Changing the aperture values one can select "tails" part of the initial distribution. This approach demonstrated its advantages and flexibility. As an example on the Figure. 1 the initial and current phase distribution functions are demonstrated. On the pictures a) - d) one can see the images of the initial function for some moment: on the part a) - the image of total distribution function, on the part b) - the image of tails particles and on the part c) - the image of the intermediate particles, on the part d) - the image of the central core particles. We should note that for most distribution function the core particles give the corresponding contribution to halo. The most extreme particles from the tail part remain in halo if they reach it once. One can see on the presented pictures different phases of halo formation for two parts of initial beam state: the first is evaluated from central part which is small enough and the second is evaluated from particles which form so-called "tail" of distribution function which can be defined using the virtual scraper concept.

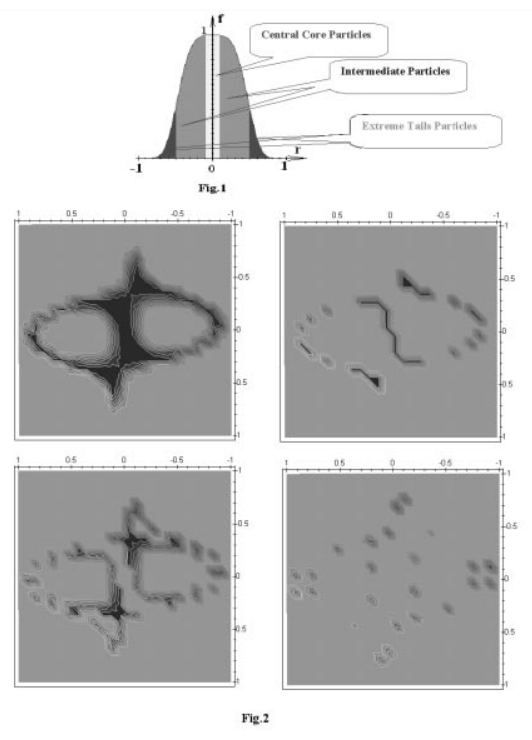

Figure 1: The initial model distribution function and 3Dcontourplots for different parts of the initial distribution

\section{REFERENCES}

[1] W.Lysenko, Z.Parsa Beam Matching and Halo Control. Preprint LA-UR-97-1712,

[2] Andrianov S.N. A Matrix Representation of the Lie Algebraic Methods for Design of Nonlinear Beam Lines, Proc. of the 1996 Comput. Acc. Phys. Conf. - CAP'96, Sept. 24-27, Williamsburg, Virginia, USA, eds. J.J.Bisognano, A.A.Mondelli, AIP Conf. Proc. 391, NY, 1997, pp.355-360.

[3] Andrianov S.N. Nonlinear Dynamics of Particle Beams with Space Charge, in: Proc. of the 9th Intern. Conf. "Computational Modelling and Computing in Physics", September 16-21, 1996, D5, 11-97-112, pp.55-60, Dubna, 1997.

[4] Andrianov S.N., High-Order Optics with Space Charge: Analytical Approach, Proc. of the Sixth European Part. Acc. Conf. - EPAC-98, Stockholm, 22-26 June 1998, Inst. of Phys. Publ., Bristol, UK, pp.1091-1093.

[5] Andrianov S.N., Edamenko N.S. Some Problems of Halo Formation in Beamlines, Abstrs. of the Fifth Intern. Workshop on Beam Dynamics \& Optimization - BDO'98, St.Petersburg, 29 June - 3 July 1998, p.12.

[6] Andrianov S.N. Dynamic Modeling Paradigm and Computer Algebra, Proc. of the Int.Conf. on Comput. Modeling and Computing in Physics, Dubna, Russia, 16-21 Sept., 1996, Dubna, 1997, pp.60-64. 